ANNALES

POLONICI MATHEMATICI

$92.1(2007)$

\title{
Results on weighted sharing of values for meromorphic functions
}

\author{
by XiAO-Min Li (Qingdao) and Hong-Xun Yi (Jinan)
}

\begin{abstract}
We prove some results on uniqueness of functions with three shared values. Our results improve those given by H. X. Yi, I. Lahiri, T. C. Alzahary \& H. X. Yi, and other authors.
\end{abstract}

1. Introduction and main results. In this paper, by meromorphic functions we shall always mean meromorphic functions in the complex plane. We adopt the standard notations in the Nevanlinna theory of meromorphic functions as explained in [3], [7], [8]. It will be convenient to let $E$ denote any set of positive real numbers of finite linear measure, not necessarily the same at each occurrence. For any nonconstant meromorphic function $h(z)$, we denote by $S(r, h)$ any quantity satisfying

$$
S(r, h)=o(T(r, h)) \quad(r \rightarrow \infty, r \notin E) .
$$

Let $f$ and $g$ be two nonconstant meromorphic functions and let $a$ be a complex number. We say that $f$ and $g$ share the value a $C M$ provided that $f-a$ and $g-a$ have the same zeros with the same multiplicities. Similarly, we say that $f$ and $g$ share the value a IM provided that $f-a$ and $g-a$ have the same zeros ignoring multiplicities. In addition, we say that $f$ and $g$ share $\infty C M$ if $1 / f$ and $1 / g$ share the value $0 \mathrm{CM}$, and we say that $f$ and $g$ share $\infty I M$ if $f$ and $g$ share the value 0 IM (see [11]). In this paper, we also need the following two definitions.

Definition 1.1 (see [1, Definition 1]). Let $p$ be a positive integer and $a \in \mathbb{C} \cup\{\infty\}$. Then we denote by $N_{p)}\left(r, \frac{1}{f-a}\right)$ the counting function of those zeros of $f-a$ (counted with proper multiplicities) whose multiplicities are not greater than $p$, and by $\bar{N}_{p)}\left(r, \frac{1}{f-a}\right)$ the corresponding reduced counting

2000 Mathematics Subject Classification: 30D35, 30D30.

Key words and phrases: meromorphic functions, weighted sharing of values, uniqueness.

Project supported by the NSFC (NO. A0324617) and the RFDP (Mo. 20060422049). 
function (ignoring multiplicities). We denote by $N_{(p}\left(r, \frac{1}{f-a}\right)$ the counting function of those zeros of $f-a$ (counted with proper multiplicities) whose multiplicities are not less than $p$, and by $\bar{N}_{(p}\left(r, \frac{1}{f-a}\right)$ the corresponding reduced counting function (ignoring multiplicities).

Definition 1.2 (see [4, Definition 4]). For $a \in \mathbb{C} \cup\{\infty\}$, we put

$$
\delta_{p)}(a, f)=1-\limsup _{r \rightarrow \infty} \frac{N_{p)}\left(r, \frac{1}{f-a}\right)}{T(r, f)},
$$

where $p$ is a positive integer.

In 1995, Yi proved the following theorem.

Theorem A (see [9, Theorem 4]). Let $f$ and $g$ be two distinct nonconstant meromorphic functions sharing $0,1, \infty C M$, and let $a(\neq 0,1)$ be a complex number. If

$$
N\left(r, \frac{1}{f-a}\right) \neq T(r, f)+S(r, f),
$$

then $a$ is a Picard exceptional value of $f$, and $f$ and $g$ satisfy one of the following three relations:

(i) $(f-a)(g+a-1) \equiv a(1-a)$;

(ii) $f+(a-1) g \equiv a$;

(iii) $f \equiv a g$.

In 1995, H. X. Yi and C. C. Yang proved the following theorem.

Theorem B (see [11, Theorem 5.13]). Let $f$ and $g$ be two distinct nonconstant meromorphic functions sharing $0,1, \infty$ CM. If $f$ is not a fractional linear transformation (Möbius transformation) of $g$, then

(i) $N_{0}\left(r, 1 / f^{\prime}\right)=\bar{N}_{0}\left(r, 1 / f^{\prime}\right)+S(r, f), \bar{N}\left(r, 1 / f^{\prime}\right)=\bar{N}_{0}\left(r, 1 / f^{\prime}\right)+$ $S(r, f)$, and the same identities hold for $g$;

(ii) $N_{(3}\left(r, \frac{1}{f-a}\right)=S(r, f), N_{(3}\left(r, \frac{1}{g-a}\right)=S(r, f)$;

(iii) $T(r, f)=\bar{N}\left(r, 1 / g^{\prime}\right)+N_{0}(r)+S(r, f), T(r, g)=\bar{N}\left(r, 1 / f^{\prime}\right)+N_{0}(r)+$ $S(r, f), N_{0}(r)=\bar{N}_{0}(r)+S(r, f)$

(iv) $T(r, f)=N\left(r, \frac{1}{f-a}\right)+S(r, f), T(r, g)=N\left(r, \frac{1}{g-a}\right)+S(r, f)$;

(v) $T(r, f)+T(r, g)=\bar{N}(r, 1 / f)+\bar{N}\left(r, \frac{1}{f-1}\right)+\bar{N}(r, f)+N_{0}(r)+S(r, f)$;

(vi) $N\left(r, \frac{1}{f-g}\right)=\bar{N}\left(r, \frac{1}{f-g}\right)+S(r, f)$;

where $N_{0}\left(r, 1 / f^{\prime}\right)$ (resp. $\left.\bar{N}_{0}\left(r, 1 / f^{\prime}\right)\right)$ denotes the counting function corresponding to the zeros of $f^{\prime}$ that are not zeros of $f$ and $f-1$ (resp. ignoring multiplicities), and $N_{0}(r)$ (resp. $\bar{N}_{0}(r)$ ) is the counting function of the zeros of $f-g$ that are not zeros of $g, g-1$ and $1 / g$ (resp. ignoring multiplicities), and $a(\neq 0,1)$ is an arbitrary complex number. 
Regarding Theorem A and Theorem B, it is natural to ask the following question.

QUESTION 1.1 (see [5]). Is it really impossible to relax in any way the nature of sharing any one of 0,1 and $\infty$ in Theorems A and B?

In this paper, we shall study this problem. Next we explain the notion of weighted sharing by the following definition.

Definition 1.3 (see [4]). Let $k$ be a nonnegative integer or infinity. For any $a \in \mathbb{C} \cup\{\infty\}$, we denote by $E_{k}(a, f)$ the set of all $a$-points of $f$, where an $a$-point of multiplicity $m$ is counted $m$ times if $m \leq k$, and $k+1$ times if $m>k$. If $E_{k}(a, f)=E_{k}(a, g)$, we say that $f, g$ share the value a with weight $k$.

Remark 1.1. Definition 1.3 implies that if $f, g$ share a value $a$ with weight $k$, then $z_{0}$ is a zero of $f-a$ with multiplicity $m(\leq k)$ if and only if it is a zero of $g-a$ with multiplicity $m(\leq k)$, and $z_{0}$ is a zero of $f-a$ with multiplicity $m(>k)$ if and only if it is a zero of $g-a$ with multiplicity $n(>k)$, where $m$ is not necessarily equal to $n$. Throughout this paper, we write $f, g$ share $(a, k)$ to mean that $f, g$ share the value $a$ with weight $k$. Clearly, if $f, g$ share $(a, k)$, then $f, g$ share $(a, p)$ for all integers $p, 0 \leq p<k$. Also we note that $f, g$ share a value $a$ IM or CM if and only if $f, g$ share $(a, 0)$ or $(a, \infty)$, respectively.

Recently, T. C. Alzahary and H. X. Yi proved the following result.

Theorem C (see [1, Theorem 1]). Let $f$ and $g$ be two distinct nonconstant meromorphic functions sharing $\left(a_{1}, 1\right),\left(a_{2}, \infty\right)$ and $\left(a_{3}, \infty\right)$, where $\left\{a_{1}, a_{2}, a_{3}\right\}=\{0,1, \infty\}$. Then either there exists an entire function $\gamma$ such that $f$ and $g$ are given by one of the following three expressions:
(a) $f=\frac{e^{\gamma}-1}{c-1}, \quad g=\frac{c\left(e^{\gamma}-1\right)}{(c-1) e^{\gamma}}$;
(b) $f=\frac{c-1}{e^{\gamma}-1}, \quad g=\frac{(c-1) e^{\gamma}}{c\left(e^{\gamma}-1\right)}$;
(c) $f=\frac{c\left(e^{\gamma}-1\right)}{e^{\gamma}-c}, \quad g=\frac{e^{\gamma}-1}{e^{\gamma}-c}$,

for some $c \in \mathbb{C} \backslash\{0,1\}$, or else for any $a \in \mathbb{C} \backslash\{0,1\}$, each of (i)-(vi) in Theorem B holds.

In this paper, we shall prove the following theorem, which improves Theorem C.

THEOREM 1.1. Let $f$ and $g$ be two distinct nonconstant meromorphic functions sharing $\left(a_{1}, k_{1}\right),\left(a_{2}, k_{2}\right)$ and $\left(a_{3}, k_{3}\right)$, where $\left\{a_{1}, a_{2}, a_{3}\right\}=\{0,1, \infty\}$, and $k_{1}, k_{2}$ and $k_{3}$ are three positive integers satisfying 


$$
k_{1} k_{2} k_{3}>k_{1}+k_{2}+k_{3}+2 .
$$

Then either there exists an entire function $\gamma$ such that $f$ and $g$ are as in (a), (b) or (c) of Theorem $C$ for some $c \in \mathbb{C} \backslash\{0,1\}$, or else for any $a \in \mathbb{C} \backslash\{0,1\}$, each of (i)-(vi) in Theorem $B$ holds.

From Theorem 1.1 and the conclusion (ii) in Theorem B, in the same manner as in the proof of Theorem 2 in [1] we easily deduce the following theorem, which improves Theorem A.

THEOREM 1.2. Let $f$ and $g$ be two distinct nonconstant meromorphic functions sharing $\left(a_{1}, k_{1}\right),\left(a_{2}, k_{2}\right)$ and $\left(a_{3}, k_{3}\right)$, where $\left\{a_{1}, a_{2}, a_{3}\right\}=\{0,1, \infty\}$, $k_{1}, k_{2}$ and $k_{3}$ are three positive integers satisfying $(1.1)$, and let $a(\neq 0,1)$ be a complex number. If

$$
N_{2)}\left(r, \frac{1}{f-a}\right) \neq T(r, f)+S(r, f),
$$

then $a$ is a Picard exceptional value of $f$, and there exists an entire function $\gamma$ such that $f$ and $g$ are given by one of the following three expressions:

(i) $f=a\left(1-e^{\gamma}\right), \quad g=(1-a)\left(1-e^{-\gamma}\right)$;

(ii) $f=\frac{a}{1-e^{\gamma}}, \quad g=\frac{a}{(a-1)\left(1-e^{-\gamma}\right)}$;

(iii) $f=\frac{a e^{\gamma}-a}{a e^{\gamma}-1}, \quad g=\frac{e^{\gamma}-1}{a e^{\gamma}-1}$.

From Theorem 1.2 we deduce the following corollary.

COROLlary 1.1. Let $f$ and $g$ be two nonconstant meromorphic functions sharing $\left(a_{1}, k_{1}\right),\left(a_{2}, k_{2}\right)$ and $\left(a_{3}, k_{3}\right)$, where $\left\{a_{1}, a_{2}, a_{3}\right\}=\{0,1, \infty\}, k_{1}, k_{2}$ and $k_{3}$ are three positive integers satisfying $(1.1)$, and let $a(\neq 0,1)$ be a complex number satisfying (1.2). If $\sigma(f)<\infty$, where $\sigma(f)$ (the order of $f$ ) is not a positive integer, then $f \equiv g$.

From Theorem 1.1, in the same manner as in the proof of Theorem 3 in [1], we deduce the following theorem.

THEOREM 1.3. Let $f$ and $g$ be two distinct nonconstant meromorphic functions sharing $\left(a_{1}, k_{1}\right),\left(a_{2}, k_{2}\right)$ and $\left(a_{3}, k_{3}\right)$, where $\left\{a_{1}, a_{2}, a_{3}\right\}=\{0,1, \infty\}$, and $k_{1}, k_{2}$ and $k_{3}$ are three positive integers satisfying (1.1). If $f$ is not any fractional linear transformation of $g$, then each of (i)-(vi) in Theorem B still holds.

\section{Some lemmas}

Lemma 2.1 (see [2]). Let $f$ and $g$ be two meromorphic functions sharing $0,1, \infty$ IM. Then

$$
T(r, f) \leq 3 T(r, g)+S(r, f) \quad \text { and } \quad T(r, g) \leq 3 T(r, f)+S(r, g) .
$$


REMARK 2.1. From Lemma 2.1 we see that if $f$ and $g$ share $0,1, \infty$ IM, then $S(r, f)=S(r, g)=: S(r)$.

Lemma 2.2 (see [10, Lemma 2.6]). Let $f$ and $g$ be two distinct nonconstant meromorphic functions such that $f$ and $g$ share $\left(0, k_{1}\right),\left(1, k_{2}\right)$ and $\left(\infty, k_{3}\right)$, where $k_{1}, k_{2}$ and $k_{3}$ are three positive integers satisfying (1.1). Then

(i) $\bar{N}_{(2}\left(r, \frac{1}{f}\right)+\bar{N}_{(2}\left(r, \frac{1}{f-1}\right)+\bar{N}_{(2}(r, f)=S(r)$;

(ii) $\bar{N}_{(2}\left(r, \frac{1}{g}\right)+\bar{N}_{(2}\left(r, \frac{1}{g-1}\right)+\bar{N}_{(2}(r, g)=S(r)$.

From Lemma 2.2 we get the following result.

LEMma 2.3. Let $f$ and $g$ be two distinct nonconstant meromorphic functions such that $f$ and $g$ share $\left(0, k_{1}\right),\left(1, k_{2}\right)$ and $\left(\infty, k_{3}\right)$, where $k_{1}, k_{2}$ and $k_{3}$ are three positive integers satisfying (1.1), and let

$$
\begin{aligned}
\frac{f-1}{g-1} & =h_{1}, \\
\frac{f}{g} & =h_{2} .
\end{aligned}
$$

Then

$$
\bar{N}\left(r, 1 / h_{j}\right)+\bar{N}\left(r, h_{j}\right)=S(r) \quad(j=1,2) .
$$

REMARK 2.2. Let

$$
h_{0}=h_{1} / h_{2}
$$

Then from Lemma 2.2 we get

$$
T\left(r, h_{j}^{\prime} / h_{j}\right)=S(r) \quad(j=1,2) \quad \text { and } \quad T\left(r, h_{0}^{\prime} / h_{0}\right)=S(r) .
$$

\section{Proof of theorems}

Proof of Theorem 1.1. We discuss the following six cases.

CASE 1 . Suppose that $f$ and $g$ share $\left(0, k_{1}\right),\left(1, k_{2}\right)$ and $\left(\infty, k_{3}\right)$. Since $f \not \equiv g$, from Lemma 2.3 we have $(2.1)$ and $(2.2)$, where $h_{1} \not \equiv 1, h_{2} \not \equiv 1$ and $h_{0} \not \equiv 1$. Combining (2.1), (2.2) and (2.4) we deduce

$$
\begin{aligned}
& f=\frac{h_{1}-1}{h_{0}-1}, \\
& g=\frac{h_{1}^{-1}-1}{h_{0}^{-1}-1} .
\end{aligned}
$$

We discuss the following four subcases.

SuBCASE 1.1. Suppose that

$$
h_{0} \equiv c,
$$


where $c(\neq 0,1)$ is a complex number. Then from (2.1), (2.2), (2.4) and (3.3) we get

$$
\frac{g}{g-1} \equiv \frac{c f}{f-1}
$$

Since $f$ and $g$ share 0,1 and $\infty$ IM, from (3.4) we deduce that $\infty$ is a Picard exceptional value of $f$ and $g$, and that $f$ and $g$ share 0 and 1 CM. So from (2.1) we can let

$$
h_{1}=e^{\gamma},
$$

where $\gamma$ is an entire function. From (3.1)-(3.3) and (3.5) we obtain the expression (a) in Theorem C.

SubCASE 1.2. Suppose that

$$
h_{1} \equiv c,
$$

where $c(\neq 0,1)$ is a complex number. Since $f$ and $g$ share 0,1 and $\infty$ IM, from (2.1) and (3.6) we deduce that 0 is a Picard exceptional value of $f$ and $g$, and that $f$ and $g$ share 1 and $\infty$ CM. From this, (2.1), (2.2), (2.4) and (3.6) we can let

$$
h_{0}=e^{\gamma},
$$

where $\gamma$ is an entire function. From (3.1), (3.2), (3.6) and (3.7) we obtain the expression (b) in Theorem C.

SuBCASE 1.3. Suppose that

$$
h_{2} \equiv c,
$$

where $c(\neq 0,1)$ is a complex number. Since $f$ and $g$ share 0,1 and $\infty$ IM, from (2.2) and (3.8) we deduce that 1 is a Picard exceptional value of $f$ and $g$, and that $f$ and $g$ share 0 and $\infty$ CM. From this and (2.1) we have (3.5), and from (2.4), (3.1), (3.2), (3.5) and (3.8) we obtain the expression (c) in Theorem C.

SubCASE 1.4. Suppose that none of $h_{1}, h_{2}$ and $h_{0}$ are constants. Let

$$
h=\frac{h_{1}^{\prime} / h_{1}}{h_{0}^{\prime} / h_{0}}=\frac{h_{1}^{\prime} / h_{1}}{h_{1}^{\prime} / h_{1}-h_{2}^{\prime} / h_{2}} .
$$

Then from (2.1), (2.2), (2.3) and (3.9) we deduce

$$
T(r, h)=S(r) \text {. }
$$

If

$$
\frac{h_{1}^{\prime}}{h_{1}} \cdot(h-1)-h^{\prime} \equiv 0
$$

then

$$
h_{1}=c(h-1),
$$


where $c(\neq 0)$ is a complex number. From (3.10) and (3.11) we deduce

$$
T\left(r, h_{1}\right)=S(r) \text {. }
$$

Again from (3.9) and (3.11) we have

$$
\frac{h_{0}^{\prime}}{h_{0}}=\frac{c h_{1}^{\prime} / h_{1}}{h_{1}+c}=-\frac{\left(c h_{1}^{-1}+1\right)^{\prime}}{c h_{1}^{-1}+1} .
$$

By integrating both sides of (3.13) we get

$$
h_{0} \equiv \frac{d}{c h_{1}^{-1}+1},
$$

where $d(\neq 0)$ is a complex number. From (3.12) and (3.14) we get

$$
T\left(r, h_{0}\right)=T\left(r, h_{1}\right)+O(1)=S(r) .
$$

From (3.1), (3.12) and (3.15) we get $T(r, f)=S(r)$, which is impossible. Thus

$$
\frac{h_{1}^{\prime}}{h_{1}} \cdot(h-1)-h^{\prime} \not \equiv 0,
$$

from which and (3.1) we get

$$
f-h=\frac{h_{1}-h_{0} h+h-1}{h_{0}-1} .
$$

Let

$$
F=(f-h)\left(h_{0}-1\right)=h_{1}-h_{0} h+h-1 .
$$

From (3.9) and (3.17) we get

$$
\begin{aligned}
\frac{F^{\prime}}{F}-\frac{h_{1}^{\prime}}{h_{1}} & =\frac{\left(h_{1}-h_{0} h+h-1\right)^{\prime}-\frac{h_{1}^{\prime}}{h_{1}} \cdot\left(h_{1}-h_{0} h+h-1\right)}{(f-h)\left(h_{0}-1\right)} \\
& =\frac{\frac{h_{1}^{\prime}}{h_{1}} \cdot(h-1)-h^{\prime}}{f-h}
\end{aligned}
$$

from which we get

$$
\frac{1}{f-h}=\frac{\frac{F^{\prime}}{F}-\frac{h_{1}^{\prime}}{h_{1}}}{\frac{h_{1}^{\prime}}{h_{1}} \cdot(h-1)-h^{\prime}} .
$$

From (2.3), (3.10) and (3.18) we deduce

$$
\begin{aligned}
m\left(r, \frac{1}{f-h}\right) & =S(r), \\
N_{(2}\left(r, \frac{1}{f-h}\right) & =S(r) .
\end{aligned}
$$


From (2.1) and (3.2) we get

$$
\frac{f-g}{g-1}=h_{1}-1 \quad \text { and } \quad g=\frac{h_{1}-1}{h_{1}-h_{2}} .
$$

Thus

$$
\frac{g^{\prime}(f-g)}{g(g-1)}=\frac{\left(\frac{h_{2}^{\prime}}{h_{2}}-\frac{h_{1}^{\prime}}{h_{1}}\right) \cdot h_{1}+\frac{h_{1}^{\prime}}{h_{1}} \cdot h_{0}-\frac{h_{2}^{\prime}}{h_{2}}}{h_{0}-1} .
$$

On the other hand, from (3.9) and (3.18) we get

$$
(f-h) \cdot\left(\frac{h_{2}^{\prime}}{h_{2}}-\frac{h_{1}^{\prime}}{h_{1}}\right)=\frac{\left(\frac{h_{2}^{\prime}}{h_{2}}-\frac{h_{1}^{\prime}}{h_{1}}\right) \cdot h_{1}+\frac{h_{1}^{\prime}}{h_{1}} \cdot h_{0}-\frac{h_{2}^{\prime}}{h_{2}}}{h_{0}-1} .
$$

From (3.22) and (3.23) we get

$$
-\frac{h_{0}^{\prime}}{h_{0}} \cdot(f-h)=\frac{g^{\prime}(f-g)}{g(g-1)} .
$$

From (2.3), (2.5), (3.20), (3.21) and (3.24) we easily deduce

$$
\begin{aligned}
N\left(r, \frac{1}{f-h}\right) & =N_{0}(r)+N_{0}\left(r, \frac{1}{g^{\prime}}\right)+S(r), \\
N_{0}(r) & =\bar{N}_{0}(r)+S(r), \\
N_{0}\left(r, 1 / g^{\prime}\right) & =\bar{N}_{0}\left(r, 1 / g^{\prime}\right)+S(r) .
\end{aligned}
$$

From (3.27) and Lemma 2.2 we easily deduce

$$
N_{0}\left(r, 1 / g^{\prime}\right)=\bar{N}\left(r, 1 / g^{\prime}\right)+S(r),
$$

which implies (i) of Theorem B. From (3.10), (3.19) and (3.25) we easily deduce

$$
T(r, f)=N_{0}(r)+N_{0}\left(r, 1 / g^{\prime}\right)+S(r) .
$$

In the same manner as above we obtain

$$
\begin{aligned}
N_{0}\left(r, 1 / f^{\prime}\right) & =\bar{N}\left(r, 1 / f^{\prime}\right)+S(r), \\
T(r, g) & =N_{0}(r)+N_{0}\left(r, 1 / f^{\prime}\right)+S(r) .
\end{aligned}
$$

From (3.28), (3.29), (3.30) and (3.31) we get (iii) of Theorem B. Next we denote by $\bar{N}_{(k, l)}\left(r, a_{i}\right)(i=1,2,3)$ the counting function of those points in $\bar{N}\left(r, 1 /\left(f-a_{i}\right)\right)$ such that $a_{i}$ is taken by $f$ with multiplicity $k$, and such that $a_{i}$ is taken by $g$ with multiplicity $l$, and each point is counted only once. First, from Lemma 2.2 we get

$$
\bar{N}(r, g)=\bar{N}_{(1,1)}(r, g)+S(r) .
$$


On the other hand, since

$$
N(r, f-g) \leq \sum_{l \geq 1} \sum_{k \geq l} k \bar{N}_{(k, l)}(r, \infty)+\sum_{k \geq 1} \sum_{l>k} l \bar{N}_{(k, l)}(r, \infty),
$$

from (3.32) and (3.33) we easily deduce

$$
\begin{aligned}
N(r, f-g)+\bar{N}(r, g) \leq & \sum_{l \geq 1} \sum_{k \geq l} k \bar{N}_{(k, l)}(r, \infty)+\bar{N}_{(1,1)}(r, g) \\
& +\sum_{k \geq 1} \sum_{l>k} l \bar{N}_{(k, l)}(r, \infty)+S(r) \\
\leq & N(r, f)+N(r, g)+S(r) .
\end{aligned}
$$

From (3.26), (3.29), (3.34) and the second fundamental theorem we have

$$
\begin{aligned}
T(r, f)+T(r, g) \leq & T(r, f)+\bar{N}\left(r, \frac{1}{g}\right)+\bar{N}(r, g)+\bar{N}\left(r, \frac{1}{g-1}\right) \\
& -N_{0}\left(r, \frac{1}{g^{\prime}}\right)+S(r) \\
= & N_{0}(r)+\bar{N}\left(r, \frac{1}{g}\right)+\bar{N}(r, g)+\bar{N}\left(r, \frac{1}{g-1}\right)+S(r) \\
\leq & \bar{N}\left(r, \frac{1}{f-g}\right)+\bar{N}(r, g)+S(r) \\
\leq & N\left(r, \frac{1}{f-g}\right)+\bar{N}(r, g)+S(r) \\
\leq & T(r, f-g)+\bar{N}(r, g)+S(r) \\
\leq & m(r, f)+m(r, g)+N(r, f-g)+\bar{N}(r, g)+S(r) \\
\leq & m(r, f)+m(r, g)+N(r, f)+N(r, g)+S(r) \\
= & T(r, f)+T(r, g)+S(r),
\end{aligned}
$$

thus

$$
T(r, f)+T(r, g)=N_{0}(r)+\bar{N}\left(r, \frac{1}{g}\right)+\bar{N}(r, g)+\bar{N}\left(r, \frac{1}{g-1}\right)+S(r)
$$

and

$$
N\left(r, \frac{1}{f-g}\right)=\bar{N}\left(r, \frac{1}{f-g}\right)+S(r) .
$$

From (3.35) and (3.36) we get (v) and (vi) of Theorem B. Since

$$
\begin{aligned}
N\left(r, \frac{1}{f^{\prime}}\right)= & N\left(r, \frac{1}{f}\right)-\bar{N}\left(r, \frac{1}{f}\right) \\
& +N\left(r, \frac{1}{f-1}\right)-\bar{N}\left(r, \frac{1}{f-1}\right)+N_{0}\left(r, \frac{1}{f^{\prime}}\right),
\end{aligned}
$$


from (3.31), (3.35), (3.37) and the second fundamental theorem we get

$$
\begin{aligned}
2 T(r, f) \leq & N\left(r, \frac{1}{f}\right)+\bar{N}(r, f)+N\left(r, \frac{1}{f-1}\right)+N\left(r, \frac{1}{f-a}\right) \\
& -N\left(r, \frac{1}{f^{\prime}}\right)+S(r) \\
= & \bar{N}\left(r, \frac{1}{f}\right)+\bar{N}(r, f)+\bar{N}\left(r, \frac{1}{f-1}\right)+N\left(r, \frac{1}{f-a}\right) \\
& -N_{0}\left(r, \frac{1}{f^{\prime}}\right)+S(r) \\
= & \bar{N}\left(r, \frac{1}{f}\right)+\bar{N}(r, f)+\bar{N}\left(r, \frac{1}{f-1}\right)+N\left(r, \frac{1}{f-a}\right) \\
& +N_{0}(r)-T(r, g)+S(r) \\
= & T(r, f)+N\left(r, \frac{1}{f-a}\right)+S(r) \\
\leq & 2 T(r, f)+S(r),
\end{aligned}
$$

which implies that

$$
N\left(r, \frac{1}{f-a}\right)=T(r, f)+S(r) .
$$

From (3.38) we get (iv) of Theorem B.

Let $z_{0}$ be a zero of $g-a$ with multiplicity $\geq 3$. Then $z_{0}$ is a zero of $g^{\prime}(f-g)$ with multiplicity $\geq 2$. From this, (2.5), (3.20) and (3.24) we obtain

$$
2 \bar{N}_{(3}\left(r, \frac{1}{f-g}\right) \leq N_{(3}\left(r, \frac{1}{g-a}\right)-\bar{N}_{(3}\left(r, \frac{1}{g-a}\right)=S(r) .
$$

From (3.39) we deduce

$$
N_{(3}\left(r, \frac{1}{g-a}\right)=S(r) .
$$

In the same manner as above we get

$$
N_{(3}\left(r, \frac{1}{f-a}\right)=S(r) .
$$

From (3.40) and (3.41) we get (ii) of Theorem B.

Case 1 is thus completely proved.

CASE 2. Suppose that $f$ and $g$ share $\left(0, k_{1}\right),\left(\infty, k_{2}\right)$ and $\left(1, k_{3}\right)$. Let $F=f /(f-1), G=g /(g-1)$, and $b=a /(a-1)$. Then $F$ and $G$ share $\left(0, k_{1}\right),\left(1, k_{2}\right)$ and $\left(\infty, k_{3}\right)$, and $F \not \equiv G$. In the same manner as in Case 1 , we find that the conclusion of Theorem 1.1 holds for $F, G$ and $b$. From this we deduce that conclusion for $f, g$ and $a$. 
CASE 3. Suppose that $f$ and $g$ share $\left(1, k_{1}\right),\left(0, k_{2}\right)$ and $\left(\infty, k_{3}\right)$. Let $F=1-f, G=1-g$, and $b=1-a$. Then $F$ and $G$ share $\left(0, k_{1}\right),\left(1, k_{2}\right)$ and $\left(\infty, k_{3}\right)$, and $F \not \equiv G$. In the same manner as in Case 1 , we find that the conclusion of Theorem 1.1 holds for $F, G$ and $b$, and hence also for $f, g$ and $a$.

CASE 4 . Suppose that $f$ and $g$ share $\left(1, k_{1}\right),\left(\infty, k_{2}\right)$ and $\left(0, k_{3}\right)$. Let $F=(f-1) / f, G=(g-1) / g$, and $b=(a-1) / a$. Then $F$ and $G$ share $\left(0, k_{1}\right),\left(1, k_{2}\right)$ and $\left(\infty, k_{3}\right)$, with $F \not \equiv G$, and we conclude as before.

CASE 5. Suppose that $f$ and $g$ share $\left(\infty, k_{1}\right),\left(0, k_{2}\right)$ and $\left(1, k_{3}\right)$. Let $F=1 /(1-f), G=1 /(1-g)$, and $b=1 /(1-a)$. Then $F$ and $G$ share $\left(0, k_{1}\right),\left(1, k_{2}\right)$ and $\left(\infty, k_{3}\right)$, with $F \not \equiv G$, and we conclude as before.

CASE 6 . Suppose that $f$ and $g$ share $\left(\infty, k_{1}\right),\left(1, k_{2}\right)$ and $\left(0, k_{3}\right)$. Let $F=1 / f, G=1 / g$ and $b=1 / a$. Then $F$ and $G$ share $\left(0, k_{1}\right),\left(1, k_{2}\right)$ and $\left(\infty, k_{3}\right)$, with $F \not \equiv G$, and we conclude as before.

Theorem 1.1 is thus completely proved.

4. On some results of Yi and I. Lahiri. In 1995, Yi and Yang proved the following result.

Theorem D (see [11, Lemma 4.5]). Let $f$ and $g$ be two distinct nonconstant meromorphic functions sharing $0,1, \infty C M$, and let $a(\neq 0,1)$ be a complex number. Then (3.41) holds.

In 2001, I. Lahiri proved the following result.

Theorem E (see [4, Lemma 5]). Let $f$ and $g$ be two distinct meromorphic functions sharing $(0,1),(1, \infty)$ and $(\infty, \infty)$, and let $a(\neq 0,1)$ be a complex number. Then

$$
\bar{N}_{(3}\left(r, \frac{1}{f-a}\right)=S(r) .
$$

In 2003, I. Lahiri proved the following result.

Theorem $\mathrm{F}$ (see $[6$, Lemma 5]). Let $f$ and $g$ be two distinct nonconstant meromorphic functions sharing $\left(a_{1}, 1\right),\left(a_{2}, m\right)$ and $\left(a_{3}, k\right)$, where $\left\{a_{1}, a_{2}, a_{3}\right\}=\{0,1, \infty\}, m$ and $k$ are positive integers satisfying

$$
(m-1)(k m-1)>(1+m)^{2},
$$

and let $a(\neq 0,1)$ be a complex number. Then (4.1) holds.

In 2004, T. C. Alzahary and H. X. Yi proved the following result.

Theorem $\mathrm{G}$ (see [1, Theorem 4]). Let $f$ and $g$ be two distinct nonconstant meromorphic functions sharing $\left(a_{1}, 1\right),\left(a_{2}, \infty\right)$ and $\left(a_{3}, \infty\right)$, where 
$\left\{a_{1}, a_{2}, a_{3}\right\}=\{0,1, \infty\}$, and let $a(\neq 0,1)$ be a complex number. Then (3.41) holds.

From Theorem 1.1 we get the following result, which improves Theorems D-G.

THEOREM 4.1. Let $f$ and $g$ be two distinct nonconstant meromorphic functions sharing $\left(a_{1}, k_{1}\right),\left(a_{2}, k_{2}\right)$ and $\left(a_{3}, k_{3}\right)$, where $\left\{a_{1}, a_{2}, a_{3}\right\}=\{0,1, \infty\}$, $k_{1}, k_{2}$ and $k_{3}$ are three positive integers satisfying $(1.1)$, and let $a(\neq 0,1)$ be a complex number. Then (3.41) holds.

5. On some other results of Yi and I. Lahiri. In 2001, I. Lahiri proved the following result.

TheORem $\mathrm{H}$ (see $[4$, Theorem 2]). Let $f$ and $g$ be two distinct meromorphic functions sharing $(0,1),(1, \infty)$ and $(\infty, \infty)$. If $a(\neq 0,1)$ is a complex number such that $3 \delta_{2)}(a, f)+2 \delta_{1)}(\infty, f)>3$, then a and $\infty$ are Picard exceptional values of $f, 1-a$ and $\infty$ are Picard exceptional values of $g$, and $(f-a)(g+a-1) \equiv a(1-a)$.

In 2004, T. C. Alzahary and H. X. Yi proved the following result.

Theorem K (see [1, Theorem 5]). Let $f$ and $g$ be two distinct nonconstant meromorphic functions sharing $\left(a_{1}, 1\right),\left(a_{2}, \infty\right)$ and $\left(a_{3}, \infty\right)$, where $\left\{a_{1}, a_{2}, a_{3}\right\}=\{0,1, \infty\}$, and let $a(\neq 0,1)$ be a complex number such that (1.2) holds.

(i) If $\bar{N}_{1)}(r, f) \neq T(r, f)+S(r, f)$, then a and $\infty$ are Picard exceptional values of $f, 1-a$ and $\infty$ are Picard exceptional values of $g$, and $(f-a)(g+a-1) \equiv a(1-a)$.

(ii) If $\bar{N}_{1)}(r, 1 / f) \neq T(r, f)+S(r, f)$, then a and 0 are Picard exceptional values of $f, a /(a-1)$ and 0 are Picard exceptional values of $g$, and $f+(a-1) g \equiv a$.

(iii) If $\bar{N}_{1)}(r, 1 /(f-1)) \neq T(r, f)+S(r, f)$, then a and 1 are Picard exceptional values of $f, 1 / a$ and 1 are Picard exceptional values of $g$, and $f \equiv a g$.

Proceeding as in the proof of Theorem 5 in [1], we can prove the following result, which improves Theorems $\mathrm{H}$ and $\mathrm{K}$.

THEOREM 5.1. Let $f$ and $g$ be two distinct nonconstant meromorphic functions such that $f$ and $g$ share $\left(a_{1}, k_{1}\right),\left(a_{2}, k_{2}\right)$ and $\left(a_{3}, k_{3}\right)$, where $\left\{a_{1}, a_{2}, a_{3}\right\}=\{0,1, \infty\}, k_{1}, k_{2}$ and $k_{3}$ are three positive integers satisfying (1.1), and let $a(\neq 0,1)$ be a complex number such that (1.2) holds. Then the conclusions of Theorem $K$ are valid.

From Theorem 5.1 we obtain the following corollary, which improves Theorem H. 
COROLlaRY 5.1. Let $f$ and $g$ be two nonconstant meromorphic functions sharing $\left(a_{1}, k_{1}\right),\left(a_{2}, k_{2}\right)$ and $\left(a_{3}, k_{3}\right)$, where $\left\{a_{1}, a_{2}, a_{3}\right\}=\{0,1, \infty\}$ and $k_{1}, k_{2}$ and $k_{3}$ are three positive integers such that $(1.1)$ holds. If a $(\neq 0,1)$ is a complex number such that $\delta_{2)}(a, f)>0$ and $\delta_{1)}(\infty, f)>0$, then the conclusions of Theorem $H$ still hold.

Acknowledgements. The authors want to express their thanks to the anonymous referee for his valuable suggestions and comments.

\section{References}

[1] T. C. Alzahary and H. X. Yi, Weighted sharing three values and uniqueness of meromorphic functions, J. Math. Anal. Appl. 295 (2004), 247-257.

[2] G. G. Gundersen, Meromorphic functions that share three or four values, J. London Math. Soc. 20 (1979), 457-566.

[3] W. K. Hayman, Meromorphic Functions, Clarendon Press, Oxford, 1964.

[4] I. Lahiri, Weighted sharing of three values and uniqueness of meromorphic functions, Kodai Math. J. 24 (2001), 421-435.

[5] - Weighted sharing and uniqueness of meromorphic functions, Nagoya Math. J. 161 (2001), 193-206.

[6] - - On a result of Ozawa concerning uniqueness of meromorphic functions II, J. Math. Anal. Appl. 283 (2003), 66-76.

[7] I. Laine, Nevanlinna Theory and Complex Differential Equations, de Gruyter, Berlin, 1993.

[8] L. Yang, Value Distribution Theory, Science Press, Beijing, 1993.

[9] H. X. Yi, Unicity theorems for meromorphic functions that share three values, Kodai Math. J. 18 (1995), 300-314.

[10] -, Meromorphic functions with weighted sharing of three values, Complex Variables 50 (2005), 923-934.

[11] H. X. Yi and C. C. Yang, Uniqueness Theory of Meromorphic Functions, Science Press, Beijing, 1995.

Department of Mathematics

Ocean University of China

Qingdao, Shandong 266071

People's Republic of China

E-mail: xmli01267@gmail.com
Department of Mathematics

Shandong University Jinan, Shandong 250100

People's Republic of China E-mail: hxyi@sdu.edu.cn

Received 23.12.2006

and in final form 17.3.2007 